\title{
A Systematic Literature Review about Idea Mining: The Use of Machine-Driven Analytics to Generate Ideas
}

\author{
Workneh Y. Ayele ${ }^{(\bowtie)}$ (D) and Gustaf Juell-Skielse (D) \\ Department of Computer and Systems Sciences, Stockholm University, Stockholm, Sweden \\ worknehadsv.su.se
}

\begin{abstract}
Idea generation is the core activity of innovation. Digital data sources, which are sources of innovation, such as patents, publications, social media, websites, etc., are increasingly growing at unprecedented volume. Manual idea generation is time-consuming and is affected by the subjectivity of the individuals involved. Therefore, the use machine-driven data analytics techniques to analyze data to generate ideas and support idea generation by serving users is useful. The objective of this study is to study state-of the-art machine-driven analytics for idea generation and data sources, hence the result of this study will generally serve as a guideline for choosing techniques and data sources. A systematic literature review is conducted to identify relevant scholarly literature from IEEE, Scopus, Web of Science and Google Scholar. We selected a total of 71 articles and analyzed them thematically. The results of this study indicate that idea generation through machine-driven analytics applies text mining, information retrieval (IR), artificial intelligence (AI), deep learning, machine learning, statistical techniques, natural language processing (NLP), NLP-based morphological analysis, network analysis, and bibliometric to support idea generation. The results include a list of techniques and procedures in idea generation through machine-driven idea analytics. Additionally, characterization and heuristics used in idea generation are summarized. For the future, tools designed to generate ideas could be explored.
\end{abstract}

Keywords: Idea mining · Idea generation · Idea elicitation · Text mining ·

Machine learning $\cdot$ Machine-driven analytics $\cdot$ Computer-assisted creativity

\section{Introduction}

The elicitation and evaluation of ideas is the core activity of innovation in organizations striving to remain competitive in the global economy. Idea generation, as a creative process, involves human judgment. Also, idea generation is the source of creativity and innovation [1]. The volume of data generated from different sources is increasing at an unprecedented rate. For example, social media, patents, and publications are increasingly growing. Organizations create large volumes of data, yet they usually fail to detect valuable ideas that could lead to innovation [2]. Besides, according to [3], research publications are growing at an unprecedented pace. However, it is hard to generate ideas. 
An idea is an abstract concept that is open to interpretations. Thorleuchter et al. referred to ideas as a piece of new and useful text phrase consisting of domain-specific terms from the context of technological language usage rather than unstandardized colloquial language [4]. On the other hand, Liu et al. defined an idea as a pair of problemsolution [5]. In this paper, we refer to "idea" as: "a sentence or text phrase describing novel and useful information through expressing possible solution(s) to current problems."

Idea generation could be done by finding analogies in diverse domains, yet eliciting relevant ideas is difficult [6]. Furthermore, ideas could be generated from diverse sources, as presented in later chapters. Idea mining, which was proposed by [4] in 2010, uses query text and textual data and apply distance-based similarity measures, unsupervised machine learning, to elicit ideas. However, several techniques in literature used other techniques and heuristics; refer to Sect. 2, Related Research and Sect. 4, Results and Analysis. Hence, we refer to these techniques as machine-driven idea generation. Research regarding idea generation supported by computer-aided or machinedriven analytics techniques is fragmented. Besides, to the best of the author's knowledge, machine-driven idea generation probably dates back to around the year 2010 refer to Sect. 2.2.

In this paper, machine-driven analytics to generate ideas uses not only idea mining, which uses text mining, machine learning, and Information Retrieval (IR), but also bibliometric analysis, NLP-enabled morphological analysis, social network analysis etc. applied on textual data to generate ideas through the use of computers. Machine-driven analytics is helpful in making sense from the increasingly accumulated digital data [7]. Generating ideas from computer-generated patterns obtained from textual data needs analytical reasoning. Endert et al. combined machine learning and visual analytics for sense-making and analytical reasoning [8]. For example, machine-driven analytics supports decision-making, which demands reasoning [9, 10]. Similarly, [11] referred to the analysis of textual data using NLP as machine-driven text analytics, and [12] referred to it as machine-driven data analytics.

This paper aims to answer the following research question: Which type of machinedriven analytics and data types are used to generate ideas? This paper also explores the state-of-the-art machine-driven idea generation, enlightens the industry and academia to promote innovation, and suggests future research directions. Therefore, a systematic literature review (SLR) over 15 years on selected papers is done. In this paper, a detailed description of mining techniques for idea generation is not presented. Hence, we advise readers from the industry to use this paper as a guideline for choosing techniques and data sources, and readers from academia to explore referred techniques as an inspiration to learning and future research possibilities. This article has six Sections: Related Research, Methodology, Results and Analysis, Discussions and Limitations, and finally Conclusions.

\section{Related Research}

This paper focuses on ideas generated through machine-driven analytics using digital data sources. Thus, potential digital sources of ideas are patents, scholarly literature [13, 14], social media [15], reports, the internet, and documents [13]. Yet, ideas could also 
be generated using crowdsourcing [2], brainstorming [16, 17], crowdfunding [18], etc. A network of experts [19] is also considered a source of ideas.

\subsection{Machine-Driven Analytics for Idea Generation}

The research findings of most machine-driven idea generation techniques either overlap with each other or use different names to refer to the same purpose. For example, in computer science, text mining combines data mining, knowledge management, IR, NLP, and machine learning [20]. Besides, machine learning deals with the study of algorithms that learn from experience and improve its performance [21]. Deep learning is part of machine learning that deals with algorithms designed by the inspiration of the human brain's function and structure [22]. Similarly, topic modeling is part of machine learning, which allows the elicitation of hidden topics from textual data [23].

Also, statistics and analytics techniques are used to generate ideas, in addition to, bibliometric and morphological analysis. Similarly, Social Network Analysis (SNA), which is an interdisciplinary work with concepts established in social theory and application, formal mathematics, statistics, and computing methodology is also used [24]. Likewise, bibliometric is a quantitative assessment of academic publications using statistical methods [25]. Authors use text mining [13, 16], data mining [26], AI [17], NLP [6, 13], SNA [27], bibliometric [28], Information Retrieval (IR) [6], deep learning [29], machine learning and experts' feedback [2], machine learning [13, 30], statistical analysis [13], idea mining [4], and topic modeling [31, 32] to generate ideas.

\subsection{Previous Research}

Authors, [33-37] claim that idea mining is introduced by Thorleuchter et al. in 2010 [4]. Thorleuchter et al. used the Euclidean distance-based similarity measuring algorithm to find solutions to queries articulated as problem statements. Ideas are then generated by finding similarities between the articulated problem queries and historical textual data, which is referred to as the distance-based algorithm applied to elicit ideas [4].

However, distance-based algorithms are not the only techniques used to deal with idea generation activities and machine-driven idea generation. For example, [38] used text mining, clustering, and visualization of experts' opinions in computer-supported brainstorming systems for idea generation. Similarly, [30] used time-series analysis and text mining on product attributes of user guides and manuals for generating new ideas in 2005. On the other hand, [39] combined the Analytic Hierarchy Process (AHP), a decision science technique, with data mining models to generate ideas using patents.

Previous literature reviews mainly focus on using distance-based similarity measuring techniques. For instance, [13] conducted a review of mining ideas from textual data, where they argued that idea mining is a recent field of study and summarized the distance-based algorithms [4]. Similarly, distance-based algorithms are used by [40] and [41]. On the other hand, [42] presented the state-of-the-art creativity supporting systems designed to generate ideas through a literature review. Thus, this article focuses on ideas generated through machine-driven analytics to improve products, services, or research-related activities. 


\section{Methodology}

To review state of the art and answer the research question, a systematic literature review (SLR) proposed by [43] and [44] is followed. As suggested by [44], the detailed guideline for conducting SLR consists of three phases: planning, conducting, and reporting. The first phase includes identifying needs, commissioning the review, articulating a research question, developing and evaluating a review protocol. The second phase includes research identification, selection of studies, quality assessment, extraction and monitoring of data, and data synthesis. The last phase includes stating dissemination mechanisms, formatting, and evaluating the report. However, commissioning, evaluating the report, and evaluating the review process are optional [44]. Additionally, to ensure that as many relevant articles as possible are selected, the snowballing technique by [45] is followed. The snowballing technique uses forward and backward searching strategies iteratively [45]. Similar to [43], the SLR methodology includes identification of relevant data sources and search strategies. It also includes selection of articles, quality assessment, data extraction, and performing synthesis based on research protocols.

\subsection{Planning the SLR}

Need for a Systematic Review. According to [13], idea mining is an interesting and new field in information retrieval. Yet, it has gained attention in the data and knowledge engineering domain [46]. Also, idea mining has been successfully used in social studies, medical, and technological domains [40]. Besides, other authors claim that idea mining is introduced by [4], see Sect. 2.2. The preliminary literature review revealed that machinedriven analytics uses other techniques that are not discussed in the definition of idea mining.

Specifying the Research Question. The research question is articulated to address the need: "Which type of machine-driven analytics and data types are used to generate ideas?". Hence, the main purpose of this study is to identify machine-driven analytics and digital data sources that are used for idea generation.

Inclusion and Exclusion Criteria. A pre-defined protocol is formulated to reduce the probability of researcher bias, as suggested in [44]. The inclusion and exclusion criteria are:

- The inclusion criteria used were: (1) publication year (2005-2020), (2) publication outlets (journals articles, and conference proceedings), (3) the selection of publications is based on relevance (papers including idea mining, idea generation, computerassisted methods, and ideation), (4) databases used (papers published in scientific journals, articles and books published in IEEE, Scopus, Web of Science, and Google Scholar).

- The exclusion criteria: (1) exclude articles that do not qualify the inclusion criteria listed above, (2) workshop papers, book reviews, and cover letters were removed.

- The search strings are derived from the research question.

- The synthesis of the work should be done to answer the research question. 


\subsection{Evaluation Criteria}

We used a predefined protocol to reduce reviewer bias. The protocol enabled us to identify relevant articles using inclusion and exclusion criteria. Additionally, we included peer-reviewed journals and conferences. Included papers were also assessed if they are relevant in terms of answering the research question. Therefore, the papers selected were assessed and checked if they contain machine-driven analytics techniques applied to textual datasets to extract ideas.

According to Kitchenham, a systemic error or bias happens when a researcher fails to produce the "true" result or deviates from it. An unbiased result is considered to be internality valid. On the other hand, applicability or generalizability is external validity, referred to as the study's applicability [43]. In this study, our results are based on the findings we obtained from the literature review, and the results are backed up by citations, and hence it is highly unlikely that we make systemic errors.

\subsection{Conducting the Review}

According to [44], the search process must include primary studies and the data extraction process provides information needed to answer the research question. Also, the data analysis process enables the researcher to answer the research question [44]. A snowballing approach is used to make sure that all relevant primary research articles are included. Additionally, to ensure that as many relevant papers as possible are included, the query included synonyms and equivalent terms, as suggested by [44]. The synthesis strategy followed is descriptive synthesis, where the research question is answered through a tabular summary, i.e. an illustrative, and explanatory presentation of the results.

We have been doing a preliminary literature study since January 2018 to explore the research demand. We collected articles for the SLR on December 29, 2019, and the inclusion of relevant articles was done until June 2020. The search query used was: (( (idea AND (generate OR generation OR generating OR elicitation OR elicit OR eliciting OR ideation))) AND (("natural language processing" OR nlp OR clustering OR "topic modeling" OR "information retrieval" OR "data analy*" OR "social network analysis") OR ("machine learning" OR "statistical learning" OR "supervised learning" OR "unsupervised learning") OR "data mining" OR "text mining" OR "idea mining"))).

\subsection{Reporting the Review}

A total of 1343 from IEEE, 1790 from Scopus, and 2077 from Web of Science articles were extracted. After removing duplicates, a total of 3809 articles remained. After applying inclusion and exclusion criteria and snowballing, a total of 71 articles were selected for the review. The result of the SLR is presented in the next Chapter. The main objective of this study is to explore machine-driven analytics techniques applied fully or partly to generate ideas and data sources used. Besides, this study explores major heuristics for idea generation applied in machine-driven techniques. The evaluation of the systematic literature review is conducted in view of the objective of the research. 


\section{Results and Analysis}

In this chapter, the result of the SLR is presented. From the SLR, we understood that idea generation using machine-driven analytics is based on underlying assumptions related to the way ideas are characterized. Therefore, in this chapter, data sources, features characterizing the availability of ideas, machine-driven idea mining techniques are presented. The SLR process is evaluated based on the predefined-protocol and relevance.

\subsection{Data Sources}

In this section, data sourced for ideas found in selected publications are presented. Seven different types of data sources appeared only once in selected publications, and these data sources are product descriptions, Wikipedia, sensor data, patents and web, webblog and documents, web-documents and publications, and patents and publications. The rest of the data sources that appeared in at least two publications are illustrated in Fig. 1 below.

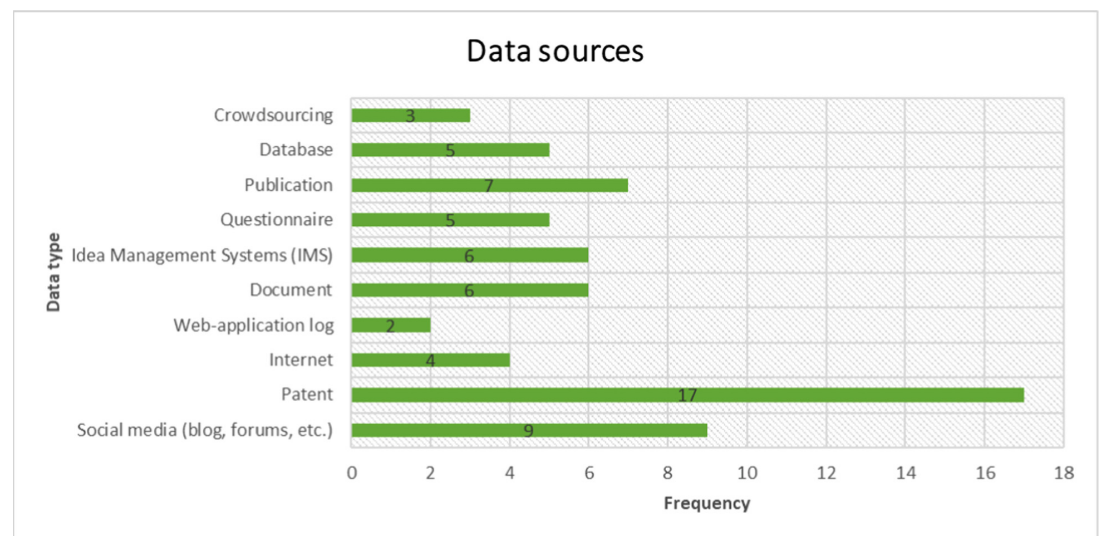

Fig. 1. Data sources that appeared in at least two publications where the labels on the bars represent frequency.

\subsection{Characterization of Textual Data for Idea Extraction}

The data sources used for idea extraction include unstructured textual data such as blog posts, social media posts, research publications, etc. which are available in a humanreadable format. The characterization of features for identifying ideas are underpinned by assumptions made regarding idea extraction. These assumptions indicate that ideas hidden within textual datasets could be extracted by examining and analyzing the nature of terms or words they contain. For example, textual data extracted from social media was used by [47] to generate creative ideas through the use of suggestive terms, such as "I think", "the solution is", "I suggest", etc. [47]. 
Phrases. Ideas are expressed in phrases, that is, terms containing $\mathrm{N}$-grams ( $\mathrm{N}$ number of terms). Hence, the elicitation of ideas could employ Part-Of-Speech (PoS) tagging to extract $\mathrm{N}$-grams, phrases with $\mathrm{N}$-words, then apply a technique to assist experts identify new and useful ideas, e.g. [48] used N-gram and Bibliometric, [49] used Ngram to extract concepts and established semantic relationship for idea generation in morphological analysis.

Problem-Solution Pairs. The assumption that problems and solutions co-exist demands for techniques to exploit these patterns using techniques such as similaritybased clustering, e.g. distance-based similarity measures, to elicit solutions to problems. For example, phrases ( $\mathrm{N}$-grams) describing problems and solutions are used to find inspirational ideas [5]. Suggestive terms, as described above, can be used to elicit association with other terms by applying association mining to discover problem-solution patterns [47].

Analogy-Based (Inspiration) Idea Generation. Analogical reasoning enables idea elicitation where ideas are generated using inspirations as analogies or solutions designed to solve similar problems. To identify inspirational stimuli during the idea generation process [50] applied Latent Semantic Analysis (LSA) for identifying the extent of semantic similarity between a database containing design examples and current work being done by participants using cosine similarity and participants rating. The author in [51] applied (text mining) cosine similarity between F-term, technical attributes to classify patent documents, and patent document to select a target technology for suggesting new technology ideas. Similarly, [52] indicated that out-of-domain knowledge, where they referred to it as distant analogies, stimulates idea generation. Also, pioneers benefit from using analogies to generate innovative ideas through searching for analogies, IR [52]. Hence, [53] applied analogical designing, clustering of patents having similar design cases, to prompt new product design idea generation [53].

Trend based or Time-Series Analysis-Based Idea Generation. Topic evolutions are generated using dynamic topic modeling using scholarly articles, and succeeding statistical time-series analysis and visualizations enable the elicitation of research and innovation ideas [32]. Similarly, [30] used text mining and time-series analysis on product attributes of user guides and manuals for generating new ideas.

\subsection{Idea Mining Techniques}

Through the use of the SLR, major machine-driven analytics for idea generation techniques are identified as illustrated in Table 1.

\subsection{Summary of Identified Idea Generation Techniques Using Idea Mining}

As discussed in Sect. 2.2, idea mining was introduced by [4], where distance-based similarity measures were used to identify new ideas. However, in this study, we have also identified that visualization, dimension reduction, NLP-driven morphological analysis, 
Table 1. List of machine-driving analytics techniques for idea generation, where Idea Type - P: Product, G: General, Pr: Process, M: Marketing, PD: Product Design, T: New Technological Idea, R: Research, C: Concept, S: Service.

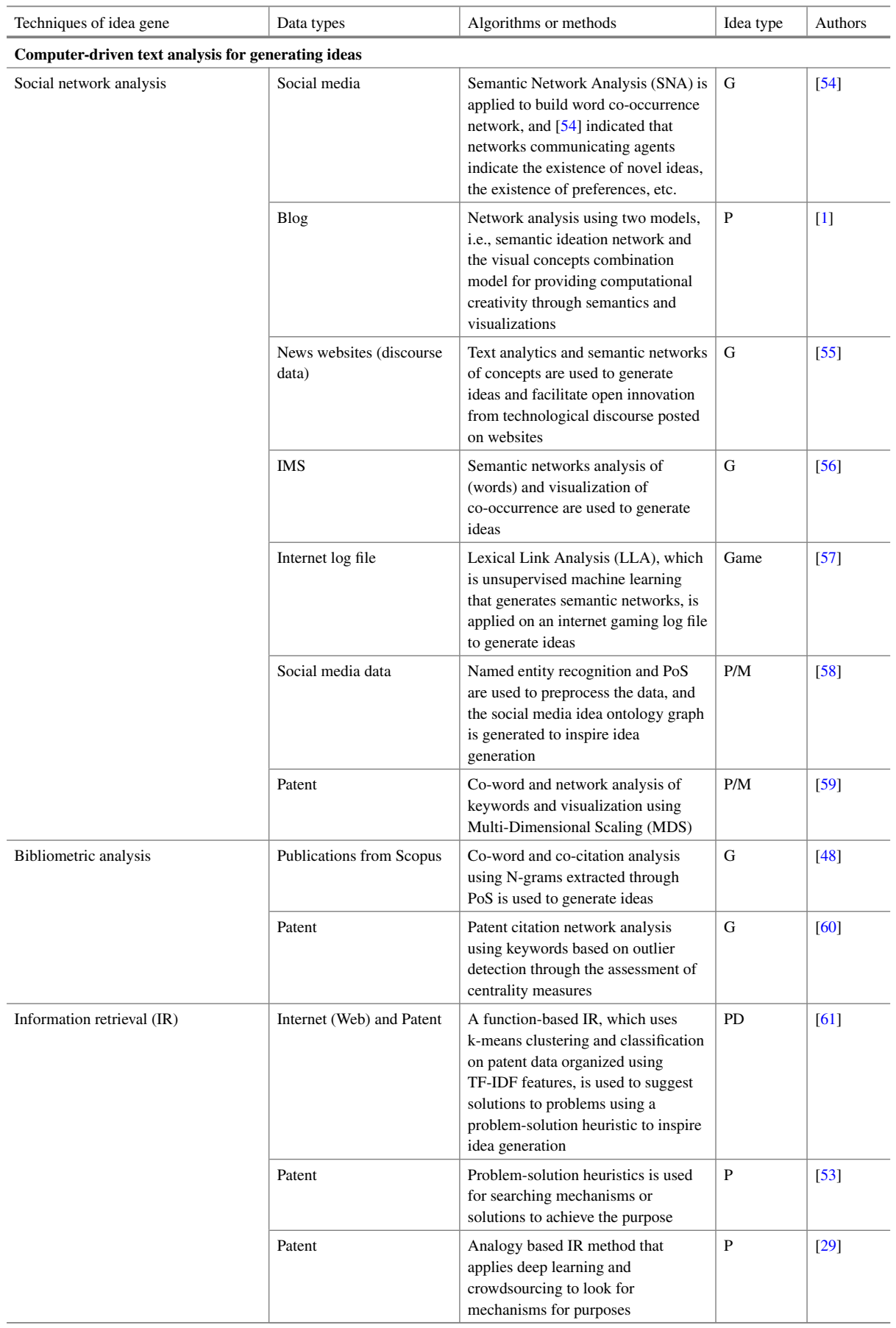


Table 1. (continued)

\begin{tabular}{|c|c|c|c|c|}
\hline Techniques of idea gene & Data types & Algorithms or methods & Idea type & Authors \\
\hline & database (product design) & $\begin{array}{l}\text { Using Big data and applying Latent } \\
\text { Semantic Analysis (LSA) for idea } \\
\text { generation }\end{array}$ & PD & {$[62]$} \\
\hline & $\begin{array}{l}\text { Social media data } \\
\text { crowdsourcing }\end{array}$ & $\begin{array}{l}\text { The use of customized databases and } \\
\text { WordNet is limited as both have } \\
\text { limitations in number and variety of } \\
\text { data. Hence authors suggested the } \\
\text { use of NLP and data mining to } \\
\text { support preprocessing } \\
\text { crowd-knowledge }\end{array}$ & PD & {$[26]$} \\
\hline \multicolumn{5}{|l|}{ Supervised machine learning } \\
\hline \multirow[t]{4}{*}{ Classification } & Document (technological) & $\begin{array}{l}\text { Using a database containing } \\
\text { analogies and a text classification } \\
\text { algorithm to inspire idea generation }\end{array}$ & $\mathrm{P}$ & {$[63]$} \\
\hline & IMS-LEGO & $\begin{array}{l}\text { SVM, nearest neighbor, decision } \\
\text { tree, neural networks - applied on } \\
\text { labeled data to demonstrate } \\
\text { identification of ideas }\end{array}$ & PD & [64] \\
\hline & IMS-LEGO \& Beer & $\begin{array}{l}\text { SVM and Partial Least Squares } \\
\text { classifiers }\end{array}$ & $\mathrm{P} / \mathrm{Pr} / \mathrm{M}$ & [65] \\
\hline & IMS & $\begin{array}{l}\text { Sentiment analysis, term features } \\
\text { extracted sung TF-IDF. Sentiment } \\
\text { analysis using SentiWordNet for } \\
\text { extracting ideas based on probability } \\
\text { of adoptability }\end{array}$ & $\mathrm{P} / \mathrm{M}$ & [66] \\
\hline $\mathrm{k}-\mathrm{NN}$ & $\begin{array}{l}\text { IMS-DELL (Dell } \\
\text { IdeaStorm) }\end{array}$ & $\begin{array}{l}\text { Outlier detection using k-NN is } \\
\text { applied on a document term matrix } \\
\text { represented as TF-IDF using cosine } \\
\text { based distance measure }\end{array}$ & $\mathrm{P} / \mathrm{S}$ & {$[67]$} \\
\hline \multirow[t]{3}{*}{ Regression and time series analysis } & IMS & NLP and Logistic regression & $\mathrm{P}$ & [35] \\
\hline & $\begin{array}{l}\text { Document (product } \\
\text { manuals) }\end{array}$ & Vector auto-regression (VAR) model & PD & [30] \\
\hline & Publication & $\begin{array}{l}\text { Using Dynamic Topic Modeling } \\
\text { (DTM) evolution of topics is } \\
\text { identified then time series analysis is } \\
\text { used to generate ideas }\end{array}$ & $\mathrm{T} / \mathrm{R}$ & {$[32]$} \\
\hline \multicolumn{5}{|l|}{ Unsupervised machine learning } \\
\hline \multirow[t]{5}{*}{ Clustering } & Patent and web report & VSM, Cosine similarity, ORCLUS ${ }^{a}$ & $\mathrm{P}$ & [68] \\
\hline & Crowdsourcing & $\begin{array}{l}\text { EM-SVD and HDBSCAN } \\
\text { algorithms and human evaluators } \\
\text { (analogy) }\end{array}$ & $\mathrm{C}$ & [69] \\
\hline & $\begin{array}{l}\text { Web document and } \\
\text { publications }\end{array}$ & $\begin{array}{l}\text { Concept clustering using similarity } \\
\text { measures between to find concept } \\
\text { association }\end{array}$ & PD & [70] \\
\hline & Patent & $\begin{array}{l}\text { Clustering using cosine-similarity } \\
\text { applied on F-term (patent } \\
\text { classification information) and patent } \\
\text { documents }\end{array}$ & $\mathrm{T}$ & {$[51]$} \\
\hline & Patent and publication & $\begin{array}{l}\text { To identify gaps and analyze the } \\
\text { correspondence between technology } \\
\text { and science, [71] used ORCUS } \\
\text { clustering }\end{array}$ & $\mathrm{T}$ & [71] \\
\hline
\end{tabular}


Table 1. (continued)

\begin{tabular}{|c|c|c|c|c|}
\hline Techniques of idea gene & Data types & Algorithms or methods & Idea type & Authors \\
\hline \multirow[t]{5}{*}{ Association mining } & Social media forums & Apriori association mining algorithm & $\mathrm{P} / \mathrm{S}$ & {$[47]$} \\
\hline & Database (data-warehouse) & Fuzzy ARM & $\mathrm{P}$ & {$[72]$} \\
\hline & Questionnaire & Apriori association mining algorithm & $\mathrm{P} / \mathrm{M}$ & {$[73]$} \\
\hline & $\begin{array}{l}\text { Database (customer and } \\
\text { transaction data) }\end{array}$ & Apriori association mining algorithm & $\mathrm{P} / \mathrm{M}$ & [74] \\
\hline & Patent & Apriori association mining algorithm & $\mathrm{P} / \mathrm{M}$ & {$[75]$} \\
\hline \multirow[t]{4}{*}{ Dimension reduction and similar } & Patent & Principal Component Analysis(PCA) & $\mathrm{P} / \mathrm{T}$ & {$[18]$} \\
\hline & Patent & $\begin{array}{l}\text { Generative Topographic Mapping is } \\
\text { better than PCA in terms of } \\
\text { visualization according to [70] }\end{array}$ & $\mathrm{T}$ & {$[76]$} \\
\hline & Patent & NLP-LSA and outlier detection & $\mathrm{P}$ & [77] \\
\hline & Patent & $\begin{array}{l}\text { WordNet on patent data to identify } \\
\text { Subject-action-object (SOA), } \\
\text { example "(S) mobile (O) has (A) } \\
\text { battery", semantic analysis based on } \\
\text { semantic similarity, and } \\
\text { multidimensional scaling } \\
\text { visualization to detect outliers } \\
\text { whereby new technological ideas } \\
\text { could be elicited }\end{array}$ & $\mathrm{T}$ & {$[78]$} \\
\hline \multirow[t]{4}{*}{$\begin{array}{l}\text { Topic modeling co-(occurrence } \\
\text { analysis) }\end{array}$} & $\begin{array}{l}\text { IMS using online } \\
\text { crowdsourcing }\end{array}$ & $\begin{array}{l}\text { Co-occurrence analysis and } \\
\text { visualization }\end{array}$ & $\mathrm{P} / \mathrm{S}$ & [79] \\
\hline & database (product design) & Using Big data and applying LSA & PD & {$[62]$} \\
\hline & Social media & $\begin{array}{l}\text { LDA is used to identify latent } \\
\text { product topics from } \\
\text { customer-generated social media } \\
\text { data and then applying sentiment } \\
\text { analysis to measure satisfaction level }\end{array}$ & $\mathrm{P} / \mathrm{M}$ & {$[80]$} \\
\hline & Publication & $\begin{array}{l}\text { DTM to generate evolution of topics } \\
\text { and visualization of trends }\end{array}$ & $\mathrm{T} / \mathrm{R}$ & {$[32]$} \\
\hline \multicolumn{5}{|l|}{ Combined techniques } \\
\hline \multirow[t]{2}{*}{ Recommendation systems } & Publications & $\begin{array}{l}\text { PoS tagging is for detecting } \\
\text { noun-phrases within titles and } \\
\text { abstracts, through which } \\
\text { problem-solution pairs and adding } \\
\text { inverse-document-frequency to make } \\
\text { it triplets for collaborative filtering } \\
\text { algorithms where problem phrases } \\
\text { are treated as users and } \\
\text { solution-phrases are treated as } \\
\text { recommended items }\end{array}$ & G & [5] \\
\hline & Patent & $\begin{array}{l}\text { LDA and collaborative filtering to } \\
\text { recommend for exploring } \\
\text { opportunities, and visualization on } \\
\text { recommended products to identify } \\
\text { new application products }\end{array}$ & $P$ & {$[81]$} \\
\hline \multirow[t]{3}{*}{ Miscellaneous } & Questionnaire & $\begin{array}{l}\text { Apriori and Clustering using } \\
\text { K-medoid and RPglobal }\end{array}$ & $\mathrm{S}$ & {$[82]$} \\
\hline & Questionnaire & $\begin{array}{l}\text { ARM (Apriori) and Clustering } \\
\text { (K-means) }\end{array}$ & $\mathrm{P} / \mathrm{M}$ & {$[83]$} \\
\hline & $\begin{array}{l}\text { Social media and } \\
\text { computer-generated data }\end{array}$ & ARM and distance-based Clustering & $S$ & {$[84]$} \\
\hline
\end{tabular}


Table 1. (continued)

\begin{tabular}{|c|c|c|c|c|}
\hline Techniques of idea gene & Data types & Algorithms or methods & Idea type & Authors \\
\hline & Patent & $\begin{array}{l}\text { Latent Dirichlet Allocation (LDA) } \\
\text { and ARM }\end{array}$ & $\mathrm{P}$ & [85] \\
\hline & $\begin{array}{l}\text { Questionnaire and } \\
\text { interview }\end{array}$ & $\begin{array}{l}\text { Apriori, K-means clustering, } \\
\text { hill-climbing and density-based } \\
\text { DBSCAN partitioning }\end{array}$ & $P$ & [86] \\
\hline & Database of examples files & $\begin{array}{l}\text { LSA and semantic similarity } \\
\text { measure (cosine similarity) to find } \\
\text { similar solutions for a given problem } \\
\text { (analogy-based) }\end{array}$ & $\mathrm{PD}$ & [50] \\
\hline & -Questionnaire and survey & $\begin{array}{l}\text { Apriori (ARM) and C5.0 (decision } \\
\text { tree) }\end{array}$ & $\mathrm{P}$ & [87] \\
\hline & Websites (crawled) & ARM and Decision tree & $\mathrm{P}$ & [88] \\
\hline & $\begin{array}{l}\text { Questionnaire and } \\
\text { interview }\end{array}$ & $\begin{array}{l}\text { Apriori (ARM) and C5.0 (decision } \\
\text { tree) }\end{array}$ & $\mathrm{P} / \mathrm{M}$ & [89] \\
\hline & $\begin{array}{l}\text { web sources about } \\
\text { renewable energy }\end{array}$ & $\begin{array}{l}\text { The idea mining technique proposed } \\
\text { by [4] was used to generate ideas, } \\
\text { and clustering using latent semantic } \\
\text { indexing was used to create semantic } \\
\text { clusters, and finally, to classify } \\
\text { concepts, Jaccard's similarity was } \\
\text { also used to identify interdisciplinary } \\
\text { idea }\end{array}$ & G & [90] \\
\hline & Social media & $\begin{array}{l}\text { Idea generation through the } \\
\text { combination of topic modeling, } \\
\text { LDA, and sentiment analysis to } \\
\text { measure satisfaction }\end{array}$ & $\mathrm{P} / \mathrm{M}$ & [80] \\
\hline
\end{tabular}

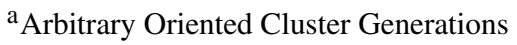

bibliometric, scientometric, and network analysis are used for idea generation. It is also found that opinion mining, idea mining, and topic signal mining are applicable for idea generation [91].

Visualization and Dimension Reduction. [77] developed a visualization tool using the NLP technique, LSA, to detect outliers for product idea generation using patent data. Also, visualization of PCA is used to detect technology gaps [18]. Unstructured data using circular layout and Force-Directed graph (CiFDAL) algorithms are applied to generate scenario graphs to generate ideas [92]. Visualization of co-occurrence graphs can be used to generate ideas [79], for example, visualization of a patent citation network of keywords is used to generate ideas by combining expert knowledge [60].

The use of NLP in combination with WordNet-based semantic similarity measure to extract terms that are subject-action-objects $(\mathrm{SAO})$ for outlier detection using a keyword relationship-based visualization algorithm called multidimensional scaling (MDS) is also applicable [78]. Outlier detection using data collected from Dell IdeaStorm and My Starbucks Idea, IMS, were used clustering to identify outliers using k-NN and TFIDF based features for generating product and service ideas [66]. The author in [78] applied WordNet on patent data to identify SAO, for example "(S) mobile (A) has (O) battery," semantic analysis based on semantic similarity, and multidimensional scaling visualization to detect outliers whereby new technological ideas could be elicited. 
Morphological Analysis is a method employed to generate ideas through breaking down a system into dimensions for the purpose of comprehensively elaborating the ins and outs of the system [93]. According to [94], the success of morphological analysis critically depends on its dimensions. However, the process of building morphological structures and values is significantly affected by subjectivity and bias, hence [94] proposed the use of WordNet, a dictionary of a lexical database consisting of a hierarchical network of words, for morphology building to establish dimensions and values. The morphological building process uses a hierarchy of words, WordNet, consisting of meronym (a semantic relation showing A is a member or part of $\mathrm{B}$, if A denotes the whole part of B, e.g., faces are used to mean people), holonym ${ }^{1}$ (a semantic relation showing a term and its relation representing it as part of whole, e.g., a finger is part of a hand), hyponym (a term which is more specific than a general term applicable to it, e.g., screwdriver is a hyponym of toolset), and hypernym (hypernym is the opposite of hyponym, e.g., color is a hypernym of green) as defined in Oxford University Press ${ }^{2}$ for dimension and value construction. Similarly, [95] demonstrated that it is possible to build a morphological matrix using Word2Vec clustering analysis and F-term for generating ideas using patent data.

Text mining is used to support the building of morphological analysis, text-driven morphological analysis, c.f. $[15,93,96]$. Geum et al. used sensor data to generate ideas [94]. The limitation of morphological analysis is that it is difficult for experts to evaluate a multitude of generated product, concept, or service ideas, therefore, [93] applied conjoint analysis, a statistical method for measuring customers' feedback among product/service idea attributes. A combination of morphological, conjoint, and citation analyses can also be used to generate new technological ideas from patents [97]. Finally, [49] used morphological analysis using Wikipedia for generating ideas using basic text analysis techniques.

Bibliometric is a quantitative assessment of academic publications using statistical methods [34]. It is possible to use publications as a data source for bibliometric analysis. Also, bibliometric combined with link mining and text mining can elicit research ideas [23]. Co-citation analysis of academic publications can be done to elicit ideas [98]. $\mathrm{Co}$-word and co-citation analysis using $\mathrm{N}$-grams extracted through PoS (Text mining, Stanford PartOfSpech) tagger from abstracts of research publications and removing noisy words, selecting $\mathrm{N}$-grams it is possible to generate ideas [48].

The Use of Enhanced IR for Searching Ideas. To aid mechanical design idea generation, [62] applied AI and big data analytics to train mechanical design using LSA for predicting the relevance of retrieved design elements for an articulated query thereby retrieving previous designs to the user. Liu et al., created a local databased through crawling the internet to extract international patent information, functional information, and patent documents through which they created function-based patent IR [94]. The function-based IR tool uses a semi-supervised machine learning to map function problem space with design problem space. They showed that function-based knowledge IR systems outperform standard IRs [61]. Similarly, [53] by applying NLP on patent

\footnotetext{
${ }^{1}$ https://en.wiktionary.org/wiki/holonym.

2 https://www.lexico.com/definition.
} 
data created an analogical source of knowledge through the development of VSM for supporting peoples creatively to generate ideas.

Patent based IR systems also support idea generation through the construction of a VSM with problem-solution space representation of patent data using crowdsourcing and deep learning [29]. The evaluation of analogy-based retrieval systems yields better performance in terms of precision and recall than traditional IR systems [29]. [63] demonstrated that a database created to store analogical information supports users in retrieving relevant analogical information that facilitates novel idea generation. [26] for product design - social media data was used, and the use of customized database and WordNet is limited as both have limitations in number and variety of data. Hence authors suggested the use of NLP and data mining to support the preprocessing of crowd-knowledge.

\section{Discussions and Limitations}

The purpose of this paper was to explore idea generation techniques. The SLR indicates that there are many techniques to support idea generation using machine-driven data analytics. Ideas are hidden within textual datasets, and these digitally available data sources are social media data, websites, different types of documents containing technical information, patents, scholarly articles, application logs, sensor data, a compilation of idea generation targeted questionnaire and interview data, databases of customer information and transactions, and documents collected during crowdsourcing events. Similarly, IMS are also sources of ideas, for example Dell IdeaStorm, My Starbucks Idea [66], LEGO, and an IMS used by a US-based Brewery [65]. The most widely used digital source of ideas in order of frequency are patents, social media, scholarly articles, IMS, questionnaires, databases, and the Internet. Also, the most widely used techniques of mining textual data for idea generation are - ARM using Apriori, clustering, a combination of $\mathrm{ARM}$ and clustering, co-occurrence analysis, dimension reduction, network analysis, and regression.

Today, machine-driven data analytics for generating ideas is demanded because it takes time to manually process large volumes of textual data. Computers need to be programmed to act like humans in order to process large volumes of data for simplifying and expediting laborious human tasks. The result of this study enabled us to define, machinedriven analytics for idea generation as "the use of digital data sources, such as textual data, to generate innovative ideas using text mining, machine learning, NLP, statistical analysis, IR, deep learning, topic modeling, social network analysis, bibliometric, NLPbased morphological analysis, and visual analytics". The most commonly used features and assumptions underpinning idea generation through machine-driven approaches are the types of words that indicate the availability of ideas, the association between words or phrases, the existence of analogies, or solutions for a given set of problems or queries, and the existence of trends and patterns. According to [47], suggestive terms such as - "I think", "The result", "I assume", etc. and their association with other terms existing in the datasets could be used to generate ideas. We also learned that analogy based searching using datasets or IR based databases expedites idea generation. Noun phrases could spur the generation of ideas. For example, text-phrases in bibliometric [48], text-phrases 
in morphological analysis [49], and text-phrases in problem-solution elicitation [5] are used for generating ideas. Time-series analysis is valuable for the elicitation of trends, temporal patterns, insights, and foresight, which in turn spur innovation. However, more works need to be done regarding the trend-driven idea generation. The result of this study could be used to support idea generation by serving as a guideline.

This study has limitations which needs to be addressed in future studies. The synthesis of selected papers is mainly focused on identification of techniques, data sources, characterizations, and heuristics. Hence, it overlooks a deeper analysis of techniques with respect to application areas. Similarly, as the purpose of this paper is mainly focused to identify techniques and data sources, a deeper analysis of aspects such as evaluation of employed techniques, heuristics involved is overlooked. For the future, it is recommended to conduct an SLR with a deeper synthesis.

\section{Conclusions}

The study of machine-driven analytics for idea generation is fragmented. For example, some consider idea generation to involve distance-based similarity to measure the semantic closeness between problem queries and historical data. In contrast, others view it as a semantic association between terms. We identified more than twenty machinedriven analytics techniques in the literature. Besides, we identified the characterization or features used for idea generation, data sources, and underlying heuristics.

Practitioners can use this study as a source of idea generation toolbox and relevant data sources. Thus, this study could summarize techniques and heuristics for idea generation for enthusiastic practitioners. It could also serve as a basis for future work in academia. Ideas are sources of innovation, and hence the result of this study is a valuable contribution to the industry, startups, accelerators, incubators, government agencies, etc.

\section{References}

1. Chen, L., Wang, P., Dong, H., Shi, F., Han, J., Guo, Y., Childs, P.R.N., Xiao, J., Wu, C.: An artificial intelligence based data-driven approach for design ideation. J. Vis. Commun. Image Represent. 61, 10-22 (2019)

2. Rhyn, M., Blohm, I., Leimeister, J.M.: Understanding the emergence and recombination of distant knowledge on crowdsourcing platforms. In: ICIS 2017 (2017)

3. Bloom, N., Jones, C.I., Van Reenen, J., Webb, M.: Are ideas getting harder to find? In: National Bureau of Economic Research (2017)

4. Thorleuchter, D., Van den Poel, D., Prinzie, A.: Mining ideas from textual information. Expert Syst. Appl. 37(10), 7182-7188 (2010)

5. Liu, H, Goulding, J., Brailsford, T.: Towards computation of novel ideas from corpora of scientific text. In: Appice, A., Rodrigues, P., Santos Costa, V., Gama, J., Jorge, A., Soares, C. (eds.) Machine Learning and Knowledge Discovery in Databases, ECML PKDD, PT II, vol. 9285, pp. 541-556 (2015)

6. Chan, J., Chang, J.C., Hope, T., Shahaf, D., Kittur, A.: SOLVENT: a mixed initiative system for finding analogies between research papers. In: Proceedings of the ACM on Human-Computer Interaction (2018) 
7. Nokia, Q., Ericsson, I., BEECube, T.V.C., Freescale, F.: IEEE GLOBECOM 2014 hosts 57th annual international conference in thriving entrepreneurial and technological center known as "the silicon hills". IEEE Commun. Mag. 53, 12 (2015)

8. Endert, A., Ribarsky, W., Turkay, C., Wong, B.W., Nabney, I., Blanco, I.D., Rossi, F.: The state of the art in integrating machine learning into visual analytics. Comput. Graph. Forum 36(8), 458-486 (2017)

9. Rendall, K., Nisioti, A., Mylonas, A.: Towards a multi-layered phishing detection. Sensors 20(16), 4540 (2020)

10. Sabeur, Z., Zlatev, Z., Melas, P., Veres, G., Arbab-Zavar, B., Middleton, L., Museux, N.: Large scale surveillance, detection and alerts information management system for critical infrastructure. In: International Symposium on Environmental Software Systems, pp. 237246. Springer, Cham (2017)

11. Bell, E., Campbell, S., Goldberg, L.R.: Nursing identity and patient-centredness in scholarly health services research: a computational text analysis of PubMed abstracts 1986-2013. BMC Health Serv. Res. 15(1), 3 (2015)

12. $\mathrm{Vu}, \mathrm{T}$.: Combating the machine ethics crisis: an educational approach. arXiv preprint arXiv: 2004.00817 (2020)

13. Alksher, M.A., Azman, A., Yaakob, R., Kadir, R.A., Mohamed, A., Alshari, E.M.: A review of methods for mining idea from text. In: 2016 Third International Conference on Information Retrieval and Knowledge Management (CAMP), pp. 88-93. IEEE (2016)

14. Rohrbeck, R.: Trend scanning, scouting and foresight techniques. In: Management of the Fuzzy Front End of Innovation, pp. 59-73. Springer, Cham (2014)

15. Kruse, P., Schieber, A., Hilbert, A., Schoop, E.: Idea mining-text mining supported knowledge management for innovation purposes. In: AMCIS (2013)

16. Itou, J., Higashi, T., Munemori, J.: Proposal and comparison of an idea generation support system presenting words for the use of scarce knowledge people. Procedia Comput. Sci. 60, 918-925 (2015)

17. Strohmann, T., Siemon, D., Robra-Bissantz, S.: brAInstorm: intelligent assistance in group idea generation. In: Maedche, A., vom Brocke, J., Hevner, A. (eds.) Designing the Digital Transformation, Desrist 2017, vol. 10243, pp. 457-461 (2017)

18. Lee, W.S., Sohn, S.Y.: Discovering emerging business ideas based on crowdfunded software projects. Decis. Support Syst. 116, 102-113 (2019)

19. Björk, J., Magnusson, M.: Where do good innovation ideas come from? Exploring the influence of network connectivity on innovation idea quality. J. Prod. Innov. Manag. 26(6), 662-670 (2009)

20. Feldman, R., Sanger, J.: The Text Mining Handbook: Advanced Approaches in Analyzing Unstructured Data. Cambridge University Press, Cambridge (2007)

21. Mitchell, T.M.: Machine learning (1997)

22. Brownlee, J.: Deep learning \& artificial neural networks in deep learning (2019)

23. Blei, D.M.: Probabilistic topic models. Commun. ACM 55(4), 77 (2012)

24. Wasserman, S., Faust, K.: Social Network Analysis: Methods and Applications, vol. 8. Cambridge University Press, Cambridge (1994)

25. Cobo, M.J., Martínez, M.Á., Gutiérrez-Salcedo, M., Fujita, H., Herrera-Viedma, E.: 25 years at knowledge-based systems: a bibliometric analysis. Knowl.-Based Syst. 80, 3-13 (2015)

26. Forbes, H., Han, J., Schaefer, D.: Exploring a social media crowdsourcing data-driven approach for innovation. In: Proceedings of the International Conference on Systematic Innovation (2019)

27. Consoli, R.: Managing creativity and innovation in web 2.0: lead users as the active element of idea generation. In: 7th ItAIS 2010, pp. 319-326 (2011) 
28. Ogawa, T., Kajikawa, Y.: Generating novel research ideas using computational intelligence: a case study involving fuel cells and ammonia synthesis. Technol. Forecast. Soc. Chang. 120, 41-47 (2017)

29. Hope, T., Chan, J., Kittur, A., Shahaf, D.: Accelerating innovation through analogy mining. In: 23rd ACM SIGKDD International Conference on Knowledge Discovery and Data Mining, KDD 2017, Part F1296, pp. 235-243 (2017)

30. Shin, J., Park, Y.: Analysis on the dynamic relationship among product attributes: VAR model approach. J. High Technol. Manag. Res. 16(2), 225-239 (2005)

31. Wang, H.C., Hsu, T.T., Sari, Y.: Personal research idea recommendation using research trends and a hierarchical topic model. Scientometrics 121(3), 1385-1406 (2019)

32. Ayele, W.Y., Juell-Skielse, G.: Eliciting evolving topics, trends and foresight about self-driving cars using dynamic topic modeling. In: Arai, K., Kapoor, S., Bhatia, R. (eds.) Advances in Information and Communication, Advances in Intelligent Systems and Computing, vol. 1129, pp. 488-509. Springer (2020)

33. Özyirmidokuz, E.K., Özyirmidokuz, M.H.: Analyzing customer complaints: a web text mining application. In: Proceedings of INTCESS14-International Conference on Education and Social Sciences, Istanbul, pp. 734-743 (2014)

34. Stoica, E.A., Özyirmidokuz, E.K.: Mining customer feedback documents. Int. J. Knowl. Eng. 1(1), 68-71 (2015)

35. Dinh, T.C., Bae, H., Park, J., Bae, J.: A framework to discover potential ideas of new product development from crowdsourcing application. arXiv preprint arXiv:1502.07015 (2015)

36. Alksher, M., Azman, A., Yaakob, R., Alshari, E., Rabiah, A.K., Mohamed, A.: Effective idea mining technique based on modeling lexical semantic. J. Theor. Appl. Inf. Technol. 96(16), 5350-5362 (2018)

37. Azman, A., Alksher, M., Doraisamy, S., Yaakob, R., Alshari, E.: A framework for automatic analysis of essays based on idea mining. In: Alfred, R., Lim, Y., Haviluddin, H., On, C.K. (eds.) Computational Science and Technology, pp. 639-648. Springer (2020)

38. Tang, X., Liu, Y., Zhang, W.: Computerized support for idea generation during knowledge creating process. In: International Conference on Knowledge-Based and Intelligent Information and Engineering Systems, pp. 437-443. Springer, Heidelberg (2005)

39. Tesavrita, C., Suryadi, K.: Decision support system for product quality development using on-line patent database. In: 36th International Conference on Computers and Industrial Engineering, ICC and IE 2006, pp. 920-928 (2006)

40. Thorleuchter, D., Van den Poel, D.: Extraction of ideas from microsystems technology. In: Advances in Computer Science and Information Engineering, pp. 563-568. Springer, Heidelberg (2012).

41. Thorleuchter, D., Van den Poel, D.: Web mining based extraction of problem solution ideas. Expert Syst. Appl. 40(10), 3961-3969 (2013)

42. Wang, K., Nickerson, J.V.: A literature review on individual creativity support systems. Comput. Hum. Behav. 74, 139-151 (2017)

43. Kitchenham, B.: Procedures for performing systematic reviews. Keele UK Keele Univ. 33(2004), 1-26 (2004)

44. Kitchenham, B., Charters, S.: Guidelines for performing systematic literature reviews in software engineering (2007)

45. Wohlin, C.: Guidelines for snowballing in systematic literature studies and a replication in software engineering. In: Proceedings of the 18th International Conference on Evaluation and Assessment in Software Engineering, p. 10 (2014)

46. Alksher, M.A., Azman, A., Yaakob, R., Kadir, R.A., Mohamed, A., Alshari, E.: A framework for idea mining evaluation. In: SoMeT, pp. 550-559 (2017) 
47. Kao, S.C., Wu, C.H., Syu, S.W.: A creative idea exploration model: based on customer complaints. In: 5th Multidisciplinary International Social Networks Conference, MISNC 2018 (2018)

48. Chen, L., Fang, H.: An automatic method for extracting innovative ideas based on the scopus ${ }^{\circledR}$ database. Knowl. Organ. 46(3), 171-186 (2019)

49. Kwon, H., Park, Y., Geum, Y.: Toward data-driven idea generation: application of Wikipedia to morphological analysis. Technol. Forecast. Soc. Chang. 132, 56-80 (2018)

50. Goucher-Lambert, K., Gyory, J.T., Kotovsky, K., Cagan, J.: Computationally derived adaptive inspirational stimuli for real-time design support during concept generation. In: ASME 2019 International Design Engineering Technical Conferences and Computers and Information in Engineering Conference (2019)

51. Song, K., Kim, K.S., Lee, S.: Discovering new technology opportunities based on patents: text-mining and F-term analysis. Technovation 60-61, 1-4 (2017)

52. Aggarwal, V., Hwang, E., Tan, Y.: Fostering innovation: exploration is not everybody's cup of tea. In: 39th International Conference on Information Systems, ICIS 2018 (2018)

53. Liu, H., Li, Y., Chen, J., Tao, Y., Xia, W.: A structure mapping-based representation of knowledge transfer in conceptual design process. Proc. Inst. Mech. Eng. Part B: J. Eng. Manuf. 234, 400-420 (2019)

54. Ghanem, A.G., Minai, A.A., Uber, J.G.: A multi-agent model for the co-evolution of ideas and communities. In: IEEE Congress on Evolutionary Computation, pp. 1-8 (2010)

55. Wehnert, P., Kollwitz, C., Daiberl, C., Dinter, B., Beckmann, M.: Capturing the bigger picture? Applying text analytics to foster open innovation processes for sustainability-oriented innovation. Sustainability (Switzerland) 10(10), 3710 (2018)

56. Toubia, O., Netzer, O.: Idea generation, creativity, and prototypicality. Mark. Sci. 36(1), 1-20 (2017)

57. Zhao, Y., Zhou, C.C., Bellonio, J.K.: Multilayer value metrics using lexical link analysis and game theory for discovering innovation from big data and crowd-sourcing. In: 2018 IEEE/ACM International Conference on Advances in Social Networks Analysis and Mining (ASONAM), pp. 1145-1151 (2018)

58. Hausl, M., Auch, M., Forster, J., Mandl, P., Schill, A.: Social media idea ontology: a concept for semantic search of product ideas in customer knowledge through user-centered metrics and natural language processing. Int. J. Comput. Inf. Eng. 11(12), 8 (2017)

59. Lee, S., Lee, S., Seol, H., Park, Y.: Using patent information for designing new product and technology: keyword based technology roadmapping. R\&D Manag. 38(2), 169-188 (2008)

60. Kim, B., Gazzola, G., Yang, J., Lee, J.M., Coh, B.Y., Jeong, M.K., Jeong, Y.S.: Two-phase edge outlier detection method for technology opportunity discovery. Scientometrics 113(1), 1-16 (2017)

61. Liu, L., Li, Y., Xiong, Y., Cavallucci, D.: A new function-based patent knowledge retrieval tool for conceptual design of innovative products. Comput. Ind. 115, 103154 (2020)

62. Steingrimsson, B., Yi, S., Jones, R., Kisialiou, M., Yi, K., Rose, Z.: Big data analytics for improving fidelity of engineering design decisions. In: 2018 SAE World Congress Experience, WCX 2018 (2018)

63. Sonal, K., Amaresh, C.: Detection and splitting of constructs of sapphire model to support automatic structuring of analogies. In: 21st International Conference on Engineering Design, ICED 2017, vol. 4, pp. 603-612. Design Society (2017)

64. Christensen, K., Norskov, S., Frederiksen, L., Scholderer, J.: In search of new product ideas: identifying ideas in online communities by machine learning and text mining. Creat. Innov. Manag. 26(1), 17-30 (2017)

65. Christensen, K., Liland, K.H., Kvaal, K., Risvik, E., Biancolillo, A., Scholderer, J., Nørskov, S., Næs, T.: Mining online community data: the nature of ideas in online communities. Food Qual. Prefer. 62, 246-256 (2017) 
66. Lee, H., Choi, K., Yoo, D., Suh, Y., Lee, S., He, G.: Recommending valuable ideas in an open innovation community: a text mining approach to information overload problem. Ind. Manag. Data Syst. 118(4), 683-699 (2018)

67. Westerski, A., Kanagasabai, R.: In search of disruptive ideas: outlier detection techniques in crowdsourcing innovation platforms. Int. J. Web Based Communities 15(4), 344-367 (2019)

68. Shen, Y.C., Lin, G.T.R., Lin, J.R., Wang, C.H.: A cross-database comparison to discover potential product opportunities using text mining and cosine similarity. JSIR 76(01), 11-16 (2017)

69. Camburn, B., Arlitt, R., Anderson, D., Sanaei, R., Raviselam, S., Jensen, D., Wood, K.L.: Computer-aided mind map generation via crowdsourcing and machine learning. Res. Eng. Des. 31, 383-409 (2020)

70. Liu, Q., Wang, K., Li, Y., Liu, Y.: Data-driven concept network for inspiring designers' idea generation. J. Comput. Inf. Sci. Eng. 20(3), 031004 (2020)

71. Wang, M.Y., Fang, S.C., Chang, Y.H.: Exploring technological opportunities by mining the gaps between science and technology: microalgal biofuels. Technol. Forecast. Soc. Chang. 92, 182-195 (2015)

72. Lee, C.K.H., Tse, Y.K., Ho, G.T.S., Choy, K.L.: Fuzzy association rule mining for fashion product development. Ind. Manag. Data Syst. 115(2), 383-399 (2015)

73. Liao, S.H., Wen, C.H.: Mining demand chain knowledge for new product development and marketing. IEEE Trans. Syst. Man Cybern. Part C (Appl. Rev.) 39(2), 223-227 (2009)

74. Liao, S.H., Hsieh, C.L., Huang, S.P.: Mining product maps for new product development. Expert Syst. Appl. 34(1), 50-62 (2008)

75. Seo, W., Yoon, J., Park, H., Coh, B., Lee, J.M., Kwon, O.J.: Product opportunity identification based on internal capabilities using text mining and association rule mining. Technol. Forecast. Soc. Chang. 105, 94-104 (2016)

76. Son, C., Suh, Y., Jeon, J., Park, Y.: Development of a GTM-based patent map for identifying patent vacuums. Expert Syst. Appl. 39(3), 2489-2500 (2012)

77. Wang, J., Chen, Y.J.: A novelty detection patent mining approach for analyzing technological opportunities. Adv. Eng. Inform. 42, 100941 (2019)

78. Yoon, J., Kim, K.: Detecting signals of new technological opportunities using semantic patent analysis and outlier detection. Scientometrics 90(2), 445-461 (2012)

79. Chen, P., Li, S., Hung, M.: Co-occurrence analysis in innovation management: data processing of an online brainstorming platform. In: 2013 Proceedings of PICMET 2013: Technology Management in the IT-Driven Services (PICMET), pp. 688-694 (2013)

80. Jeong, B., Yoon, J., Lee, J.M.: Social media mining for product planning: a product opportunity mining approach based on topic modeling and sentiment analysis. Int. J. Inf. Manag. 48, 280-290 (2019)

81. Yoon, J., Seo, W., Coh, B.Y., Song, I., Lee, J.M.: Identifying product opportunities using collaborative filtering-based patent analysis. Comput. Ind. Eng. 107, 376-387 (2017)

82. Karimi-Majd, A.M., Mahootchi, M.: A new data mining methodology for generating new service ideas. Inf. Syst. E-Bus. Manag. 13(3), 421-443 (2015)

83. Liao, S.H., Chen, Y.N., Tseng, Y.Y.: Mining demand chain knowledge of life insurance market for new product development. Expert Syst. Appl. 36(5), 9422-9437 (2009)

84. Karimi-Majd, A.M., Fathian, M.: Extracting new ideas from the behavior of social network users. Decis. Sci. Lett. 6, 207-220 (2017)

85. Feng, L., Li, Y., Liu, Z., Wang, J.: Idea generation and new direction for exploitation technologies of coal-seam gas through recombinative innovation and patent analysis. Int. J. Environ. Res. Public Health 17(8), 2928 (2020)

86. Liao, S., Chen, Y.J., Deng, M.: Mining customer knowledge for tourism new product development and customer relationship management. Expert Syst. Appl. 37(6), 4212-4223 (2010) 
87. Zhan, Y., Tan, K.H., Huo, B.: Bridging customer knowledge to innovative product development: a data mining approach. Int. J. Prod. Res. 57(20), 6335-6350 (2019)

88. Lee, C., Song, B., Park, Y.: Design of convergent product concepts based on functionality: an association rule mining and decision tree approach. Expert Syst. Appl. 39(10), 9534-9542 (2012)

89. Bae, J.K., Kim, J.: Product development with data mining techniques: a case on design of digital camera. Expert Syst. Appl. 38(8), 9274-9280 (2011)

90. Thorleuchter, D., Van den Poel, D.: Identification of interdisciplinary ideas. Inf. Process. Manag. 52(6), 1074-1085 (2016)

91. Lee, T.Y.: A study on extracting ideas from documents and webpages in the field of idea mining. J. Korean Soc. Inf. Manag. 29(1), 25-43 (2012)

92. Wang, Y., Zhang, C., Wang, W., Xu, F., Wang, H.: CiFDAL: a graph layout algorithm to enhance human cognition in idea discovery. In: 2015 IEEE International Conference on Systems, Man, and Cybernetics, pp. 1545-1550 (2015)

93. Kim, C., Lee, H.: A database-centred approach to the development of new mobile service concepts. Int. J. Mob. Commun. 10(3), 248 (2012)

94. Geum, Y., Jeon, H., Lee, H.: Developing new smart services using integrated morphological analysis: integration of the market-pull and technology-push approach. Serv. Bus. 10(3), 531-555 (2016)

95. Feng, L., Niu, Y., Liu, Z., Wang, J., Zhang, K.: Discovering technology opportunity by keyword-based patent analysis: a hybrid approach of morphology analysis and USIT. Sustainability 12(1), 136 (2020)

96. Han, M., Park, Y.: Developing smart service concepts: morphological analysis using a noveltyquality map. Serv. Ind. J. 39(5-6), 361-384 (2019)

97. Yoon, B., Park, Y.: Development of new technology forecasting algorithm: hybrid approach for morphology analysis and conjoint analysis of patent information. IEEE Trans. Eng. Manag. 54(3), 588-599 (2007)

98. Ayele, W.Y., Akram, I.: Identifying emerging trends and temporal patterns about self-driving cars in scientific literature. In: Arai, K., Kapoor, S. (eds.) Advances in Computer Vision, CVC 2019. Advances in Intelligent Systems and Computing, vol. 944. Springer, Cham (2020) 